\title{
AFRAME:
}

\section{A Video Animation Capability for Displaying FINDIF Numerical Model Output}

\author{
W. S. Little and R. A. Stephen \\ Woods Hole Oceanographic Institution \\ Woods Hole, MA 02543
}

July, 1995

Technical Memorandum

This work was carried out under ONR Grant \#N00014-90-J-1493
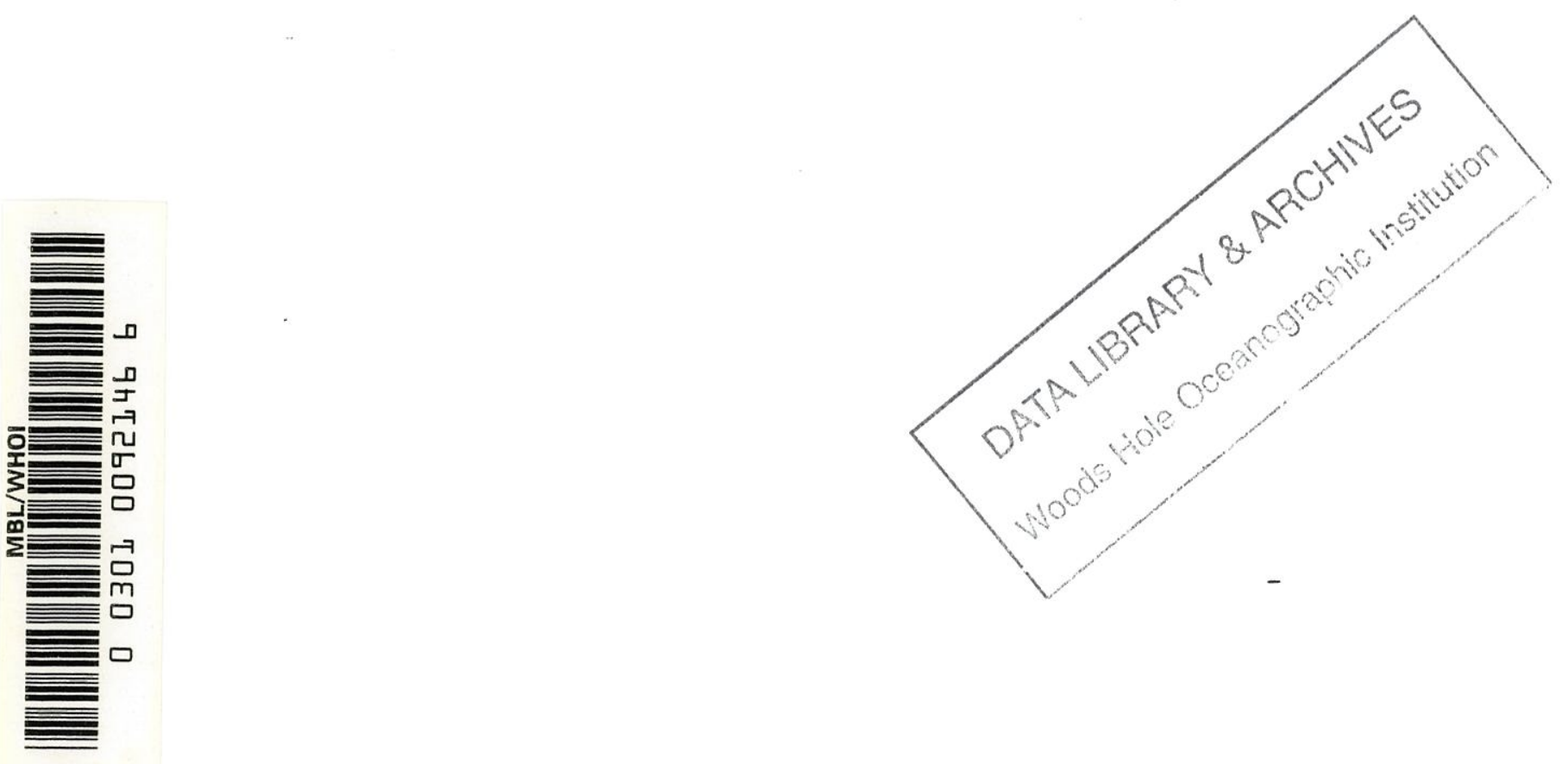


\section{Table of Contents}

Introduction 1

Background $\quad$. 1

PHASE 1: VAX/VMS FORTRAN and UNIRAS Implementation $\quad 2$

Hardware and Software Configuration $\quad 2$

Processing Steps 3

Preprocessing Script 3

FINDIF Operation from LaSpezia $\quad 7$

Phase 1 FINDIF/AFRAME Results

Early AFRAME Experiments and Modifications $\quad 7$

Standardized Frame Sizè 9

Other Phase 1 Users 9

PHASE 2: UNIX C Language and PHOTOSHOP Implementation 12

Program Conversion $\quad 12$

New Software Configuration $\quad 12$

Equipment Relocation $\quad 12$

Phase 2 FINDIF/AFRAME Results 15

Non-homogeneous Media and Higdon Boundaries $\quad 15$

Further Modifications $\quad 15$

Other Phase 2 Users 15

$\begin{array}{ll}\text { Work in Progress } & 16\end{array}$

Conclusion 16

$\begin{array}{lr}\text { Acknowledgment } & 16\end{array}$

$\begin{array}{ll}\text { References } & 17\end{array}$

Appendix 1: Memorandum proposing a WHOI computer
animation facility

Appendix 2: Selected Abekas A60 disk users $\quad 22$

Appendix 3: FINDIF Video Tape Products $\quad 22$

Figures

1. WHOI Video Animation System Hardware Configuration 4

2. WHOI Video Animation System Software Configuration 5

3. AFRAME Processing Flow Diagram (Phase 1) 6

4. Sample Video Frame: Soft Rough Seafloor, Low Beam Angle 8

5. Relationship between FINDIF Model Space and Video Frame 10

6. Sample Photoshop Overlay Frame 13

7. AFRAME Processing Flow Diagram (Phase 2) 14 


\section{AFRAME: \\ A Video Animation Capability for Displaying \\ FINDIF Numerical Model Output}

\section{Introduction}

This report describes the development of a facility for recording timevarying computer graphics on video tape. The primary purpose of the facility is to produce animation sequences of ocean and seafloor acoustic wave fields from output of the synthetic seismogram numerical model FINDIF, and to record them on convenient portable VHS video tapes.

The facility utilizes a suite of computer programs called AFRAME, and an Abekas model A60 digital video disk which is connected to the modeling computer and to broadcast quality video recording equipment.

\section{Background}

Numerical modeling of geoacoustic wave propagation has been used by Stephen $(1991,1994)$ to study acoustic bottom interaction and scattering.

A modeling program called FINDIF has been developed by Hunt, Gove, and Stephen (1983) which simulates this wave propagation by numerically solving the elastic wave equation in two dimensions, using the method of finite differences (Stephen, 1988). The model allows for the introduction of an acoustic source at some point in the model space (which is a vertical slice of ocean extending from the sea surface deep into the sea floor), and then calculates for successive time-steps the acoustic disturbances throughout the model space. As the model evolves, it produces output files comprising time series of the vertical and horizontal displacement or pressure at lattice points making up the supporting media.

At specified time-steps during execution, the model also produces a sequence of 2-D "snapshots" of various acoustic wave fields such as the compressional (pressure) and shear amplitude density, or the vertical and horizontal displacement at lattice points (see Appendix to Stephen, 1994). Viewed in graphical form on a computer display with gray-scale capabilities, these snapshots show the overall wave pattern at various time-steps.

When viewed in rapid succession, a series of snapshots provides an animated representation of the evolution of the acoustic waves during the entire model run, much like watching surface waves caused by a stone dropped in a pond (Schlieren diagrams). This time-varying graphical view of the wave fields constitutes an excellent way to qualitatively examine the overall behavior of a model run. This technique serves not only as a convenient means of illustrating the physics of the phenomena to colleagues and students, but also as a useful debugging tool during the development of the numerical algorithms used in the model. 
Because of the long execution times typically associated with FINDIF runs, it was necessary to store several snapshots in computer memory in order to play back the animation sequences in rapid succession. Since computer memory was very limited at the time this work was begun, and because graphics computers were not then easily portable, it became desirable to record the animated sequences on inexpensive VHS-format video tape.

To facilitate the transfer of FINDIF snapshot animation sequences from computer to video format, Stephen acquired in 1990 as part of the ONR Acoustic Reverberation Special Research Program (ARSRP) an Abekas Model A60 digital video disk (Appendix 1). The Abekas A60 disk is essentially a 525 Mbyte disk with a $21 \mathrm{Mbyte/second} \mathrm{transfer} \mathrm{rate,} \mathrm{of} \mathrm{the} \mathrm{variety} \mathrm{used} \mathrm{for} \mathrm{creating} \mathrm{TV}$ broadcast "spinning logos" and sports casting stop-frame replays. The A60 can store up to 750 full color computer graphics images and then play them back in random order at the very high rate required for full motion video. At the time this system was acquired, it was one of the only ways to convert high quality computer images to video format.

When the A60 disk was acquired it was interfaced to the WHOI campus Internet so that computer graphics data frames could be transferred directly to the disk from most computers on the network. The A60 was also connected to an existing Betacam broadcast quality video recorder, which in turn was connected to other video editing equipment, and ultimately to VHS recorders.

A suite of computer programs were developed for utilizing the A60 to generate video animation sequences directly from FINDIF snapshot output. The programs are now collectively referred to as AFRAME (short for Animation Frame maker). 'The suite of AFRAME software was rewritten several times to adapt it to changing input requirements. For simplicity, one can think of the development of the software as having occurred in two phases, the first of which dealt primarily with data from VAX/VMS-based models, and the second of which handled data primarily from UNIX systems.

\section{PHASE 1: VAX/VMS FORTRAN and UNIRAS Implementation}

The original implementation of the AFRAME software consisted of a collection of FORTRAN programs developed for a VAX 8800 computer at WHOI. This version of AFRAME used UNIRAS graphics software to do the actual generation of individual animation frames. The VAX used the VMS operating system.

\section{Hardware and Software Configuration}

The video equipment was located in WHOI's Clark Laboratory adjacent to the computer terminals which were used to access the modeling computers. The equipment at this time consisted of the Abekas A60 video disk, and a borrowed Betacam VCR, video sync generator, and miscellaneous video equipment (Figure 1). The A60 video output signal was aligned for Betacam recording so that it could be interfaced directly to a Sony Betacam studio-

quality VCR. The system was configured to provide for the transfer of individual composited RGB or YUV format frames of a standard size ( 720 by 486 
pixels) from the host computer to the A60 using the TCP/IP file transfer protocol (ftp) (Figure 2) .

The Phase 1 AFRAME software system essentially combined lettering and line art overlays with gray-scale bitmap images derived from snapshots of the P-wave and S-wave fields produced by the numerical model (Figure 3). FINDIF snapshots were converted to images, scaled, clipped, and positioned within each video frame using the program called FRAME. FINDIF boundary conditions were used to delineate the seafloor in the resulting images. Static graphics overlays (and a frame counter overlay) were created with the program called OVERLAY. Both FRAME and OVERLAY use UNIRAS subroutines. Frames and overlays were combined using the program MASK, and finally filtered by FILTER, using rgb-to-yuv color space conversion routines, in order to produce acceptable video frames for subsequent transfer to the A60 disk.

\section{Processing Steps}

The overall processing flow was made up of the following steps:

- $\quad$ Run FINDIF to produce a series of snapshot files.

- Use the preprocessing program OVERLAY to create the appropriate lettering and line art. ("Title" and "credits" stillframes were also created in this manner.)

- $\quad$ Run FRAME and MASK on each frame (using a command file) to produce a series of Abekas video frames on ordinary computer disk.

- $\quad$ ftp each frame file from the ordinary computer disk to the corresponding frame on the Abekas A60 video disk.

- Use the firmware-based sequencing and looping capabilities of the $A 60$ to specify a final video sequence, and then transfer the sequence to a Betacam video tape master in real time.

- Use the available video copying equipment to copy the Betacam master tape to one or more VHS tapes for use general use.

\section{Preprocessing Script}

Since the basic lettering capabilities of UNIRAS were not adequate for video purposes, the program OVERLAY used a rudimentary script-based preprocessing arrangement to provide more flexibility in the manipulation and placement of the graphics elements within each animation frame. This preprocessing allowed for the creation of bold, shadow-dropped, block lettering, wide lines, and a simple WHOI logo which were required to produce more readable, more pleasing graphics on low-resolution NTSC VHS video displays. 
Figure 1

WHOI Video Animation System

Hardware Configuration

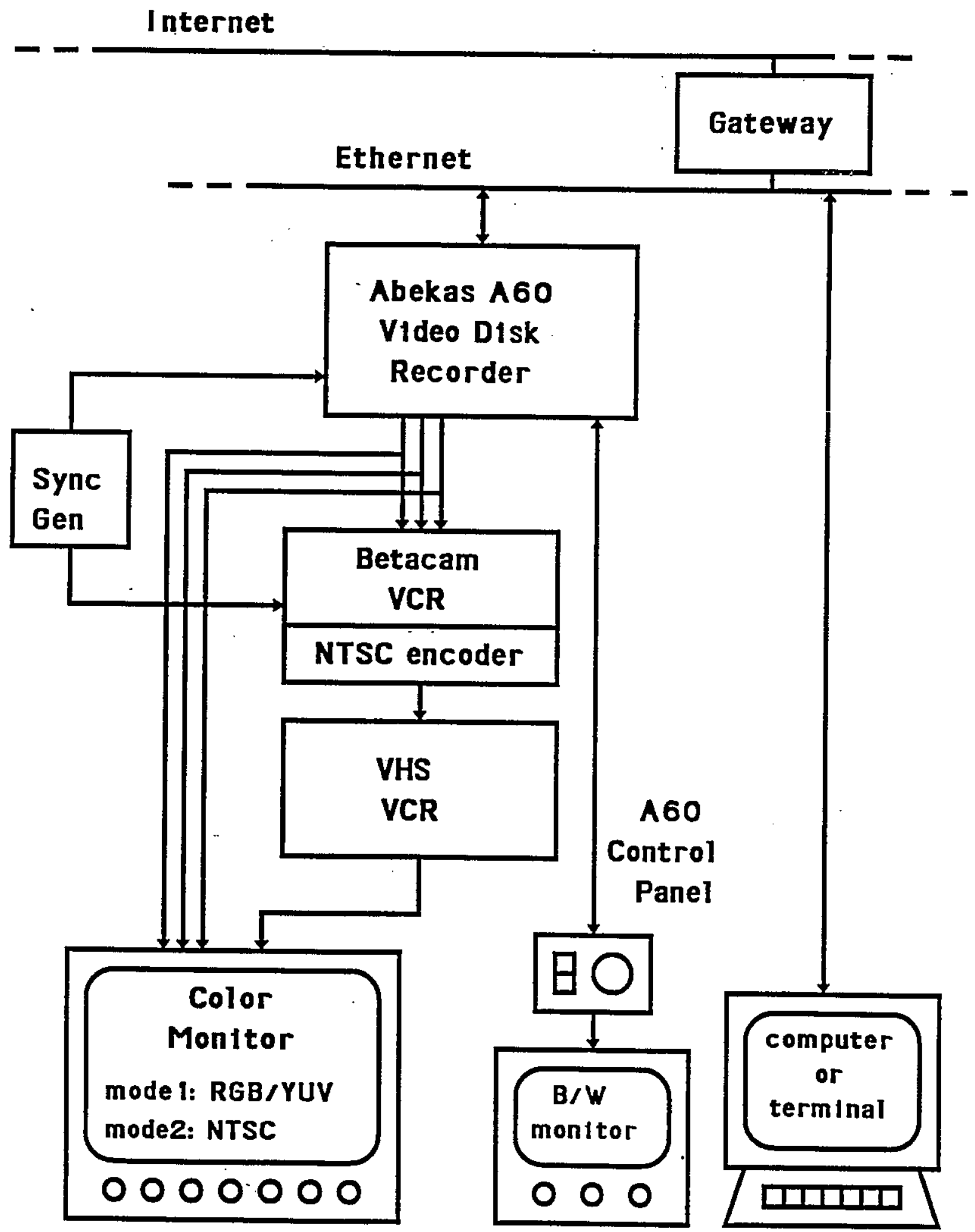


Figure 2:

WHOI VIdeo Amination System

Software Configuration
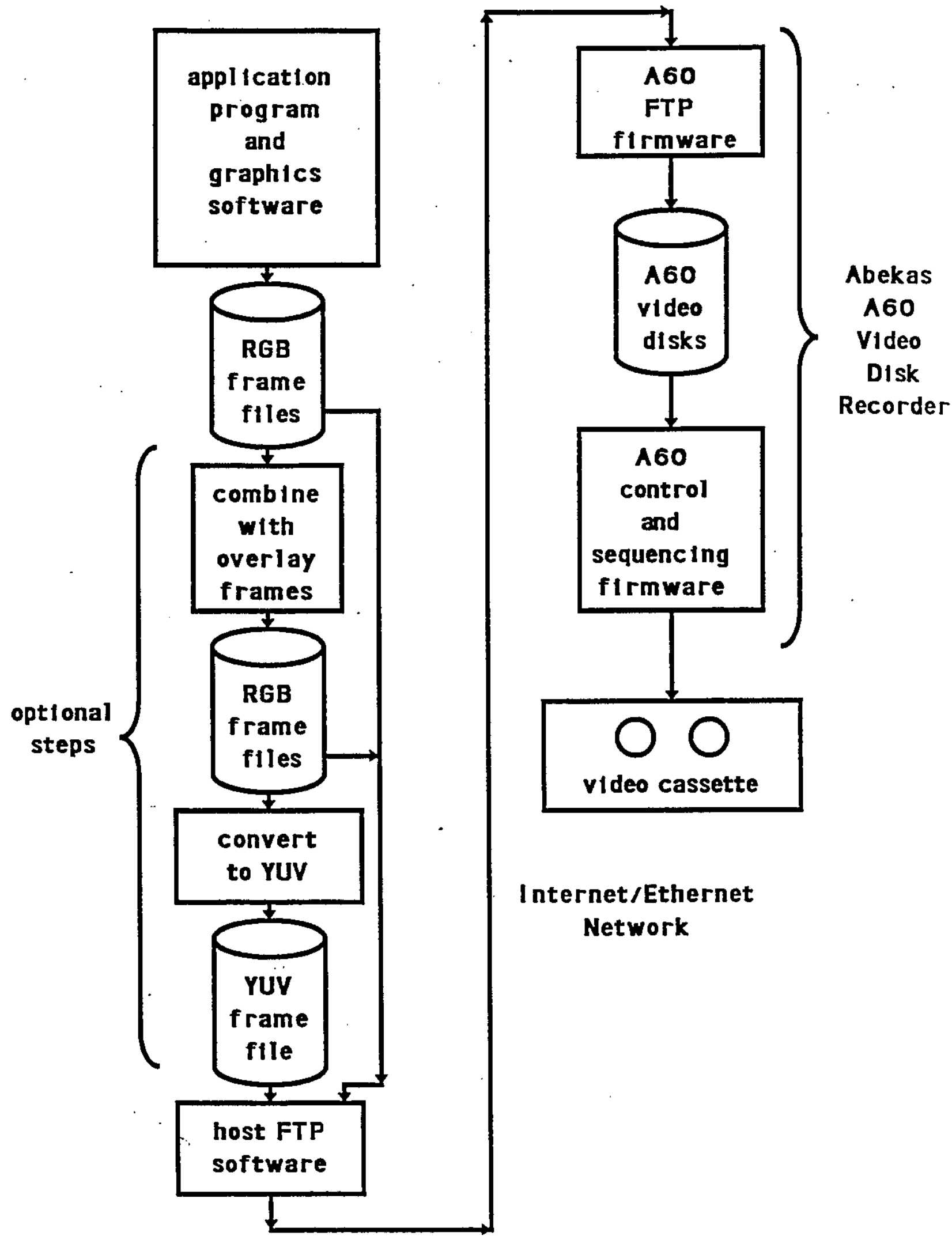

Internet/Ethernet

Network 


\section{Figure 3}

\section{AFRAME Processing Flow Diagram (Phase 1)}

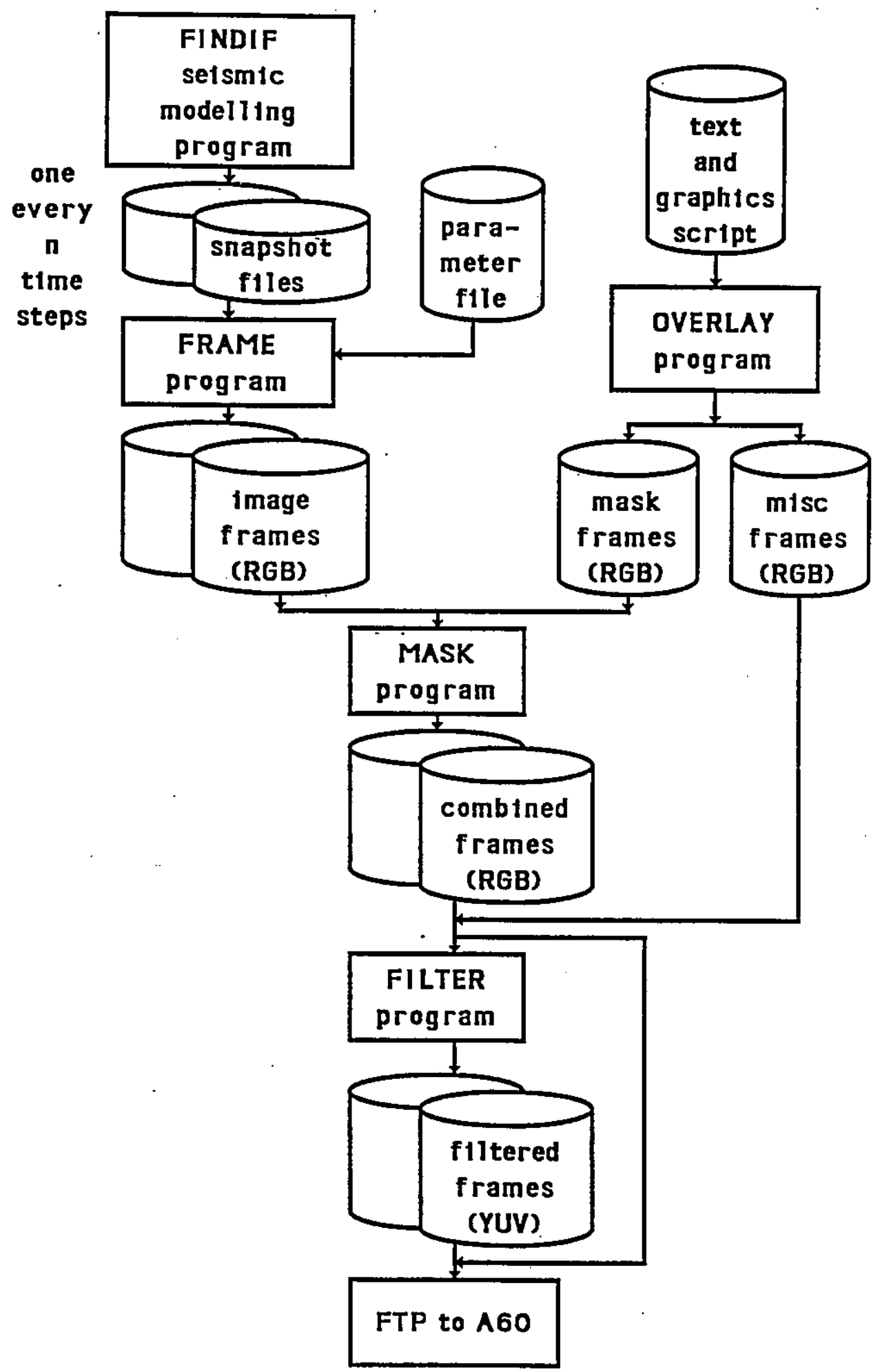




\section{FINDIF Operation from LaSpezia}

During most of the first year of operation, Stephen was a visiting scientist in residence at the NATO SACLANT Center in LaSpezia, Italy, where he conducted much of his modeling using another VAX/VMS system located in Italy. The LaSpezia VAX was not then on the Internet, which at that time did not exist in its present form. As a result, all computer-related correspondence, including significant changes to the FINDIF model code, had to be relayed to WHOI from LaSpezia by FAX or mail and then manually re-entered into the WHOI VAX.

Each revision of the LaSpezia code would then be re-run on the WHOI VAX in order to recreate the same series of output snapshots of P-wave and Swave fields which had been created in the original run. Next AFRAME would be used to produce a "draft" video tape which would be shipped to LaSpezia for editorial review. Cosmetic and substantive bugs in the draft video (as well as bugs uncovered in the original LaSpezia model run, or in the recreated WHOI model run) would be corrected, and the entire process repeated until presentation quality video sequence had been produced.

\section{Phase 1 FINDIF/AFRAME Results}

This process was used in the production of several acceptable video sequences showing, among them a suite of five model runs showing shallow angle back scattering in homogeneous water, hard and soft flat seafloors, and hard and soft rough seafloors (Figure 4). These results were presented at the ARSRP Symposium at Scripps in March, 1991 (Little, Stephen, 1991).

\section{Early AFRAME Experiments and Modifications}

During Phase 1, the original AFRAME system was modified several times in order to accept snapshots from UNIX systems (e. g., Convex C220 Minisupercomputer, Sun Workstations), deal with byte-swapping problems, accept floating point formats generated by computers from different manufacturers, and accommodate different snapshot frame sizes, orientations, sub-sampling frequencies, color scales, number of color levels, and signal thresholds.

We determined that in general gray-scale imagery was more suitable than colored imagery for showing wave propagation on VHS tapes, not only because it was less distracting and confusing, but also because the horizontal screen resolution of NTSC VHS video displays is twice as great in black-andwhite (the analog video luminance signal) as it is in color (the analog video chrominance signal ).

Experiments were undertaken to create tutorial videos in which the various components of the wave divergence and curl fields were annotated with moving pointers. In addition, some video sequences utilizing waves of various colors to show different orders of magnitude were attempted, in an effort to display a wider dynamic range of pressure and shear wave amplitudes. 


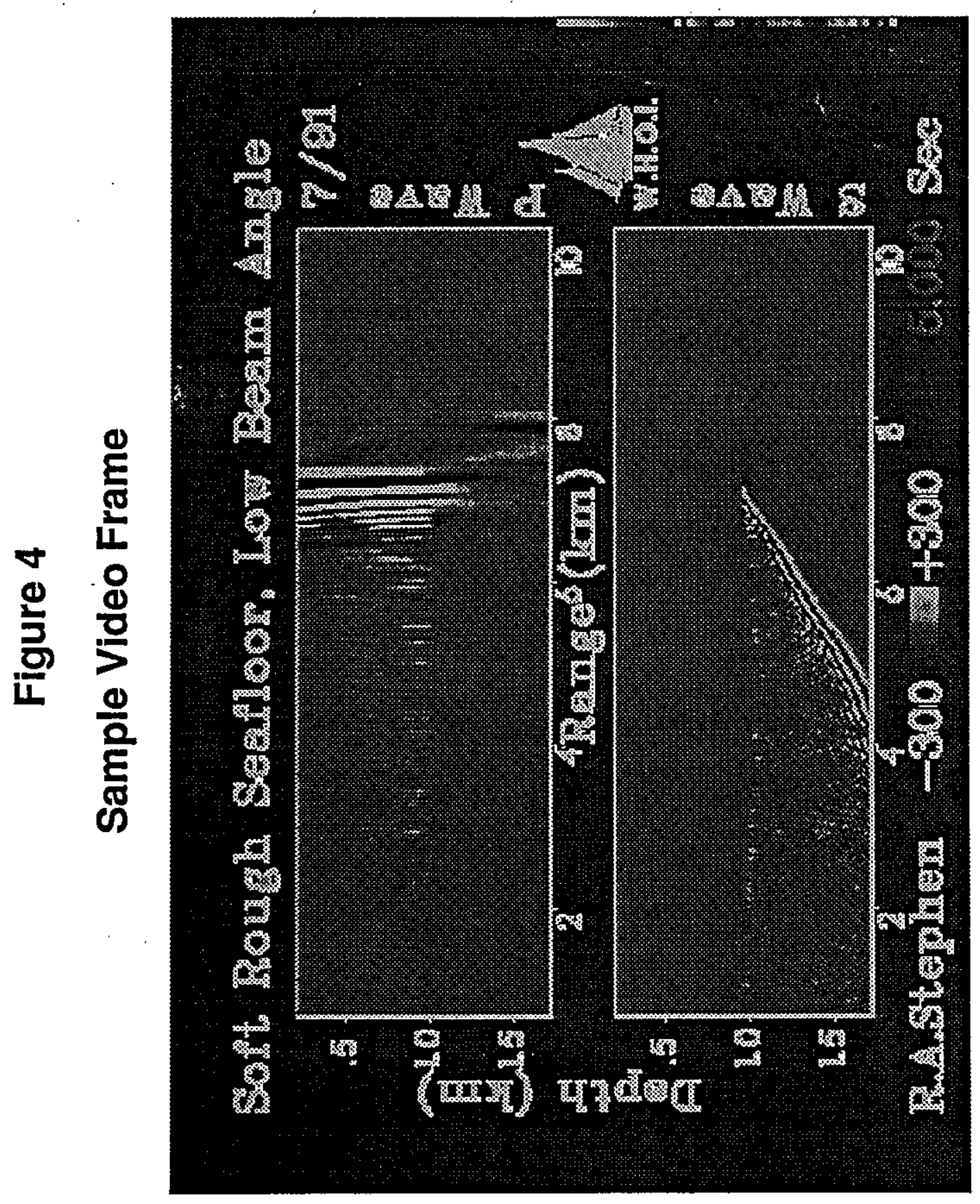


Since this work was all done prior to the age of inexpensive desktop computer-to-video equipment and widely available video filtering software, considerable effort was spent making lettering, line art and the moving waveform images "look nice" on home quality video tape. Rudimentary softwarebased anti-aliasing and color filtering were used to reduce many of the video artifacts which otherwise result from overly saturated colors ("illegal colors" in video parlance), highly abrupt hue and/or intensity changes, illegal spatial frequencies (the "striped necktie" effects), and so forth.

\section{Standardized Frame Size}

After considerable experimentation, we ultimately settled on a standard model space layout '(including absorbing boundary and hydrophone locations), a standard still frame (Spyglass) layout, and a standard video snapshot layout and sub-sampling strategy (Figure 5). The latter was chosen to allow us to show the most detailed wave propagation patterns, consistent with the reduced resolution of the final VHS video tapes.

\section{Other Phase 1 Users}

During this period, several other principal investigators were able to make use of the video system (Appendix 2). Various promotional demonstration tapes were produced showing selected uses of video animation capabilities in the field of oceanography. These were used by the institution director and by the computer center director in describing some of the research activities being conducted at WHOI using this technology. (Appendix 3 lists several videos produced during the program.) 


\section{Figure 5a \\ FINDIF Model Space}

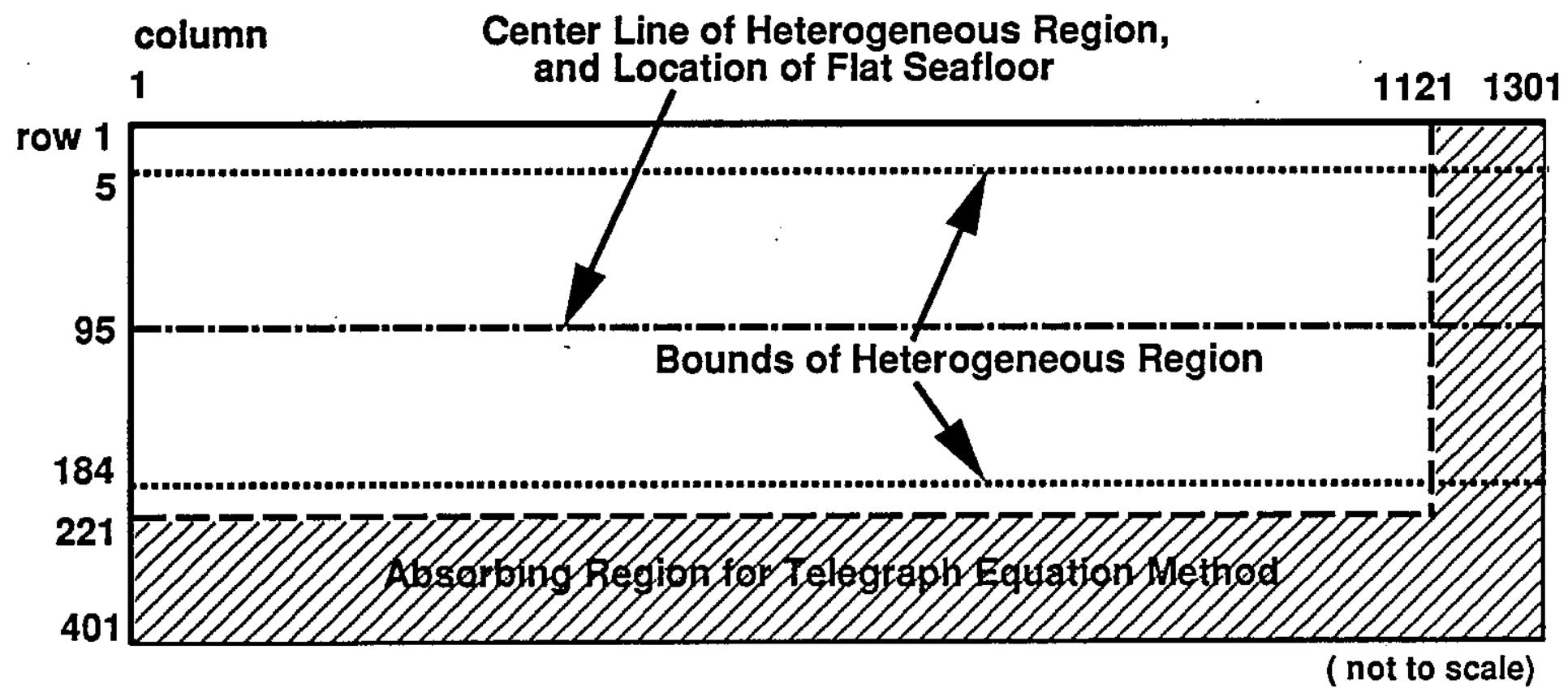

column FINDIF Parameter Summary

Figure 5b

11171

10301100

1091

$$
5
$$

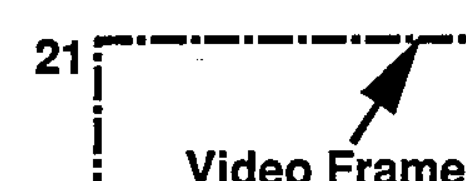

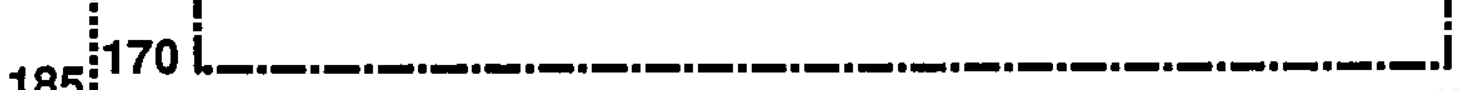

\section{Video Frame}

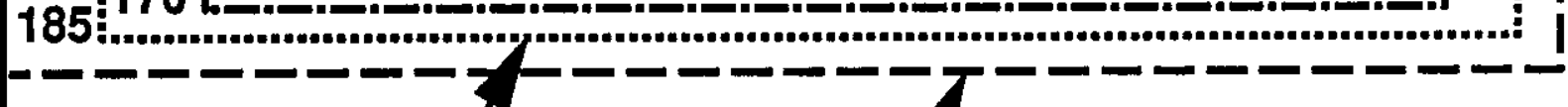

Receiver Box

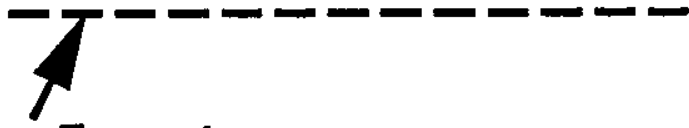

Spyglass Format

Snapshots

401

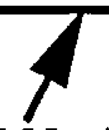

( not to scale)

FINDIF Model Space 
Figure 5c

Relationship between FINDIF

Model Space and Video Frame

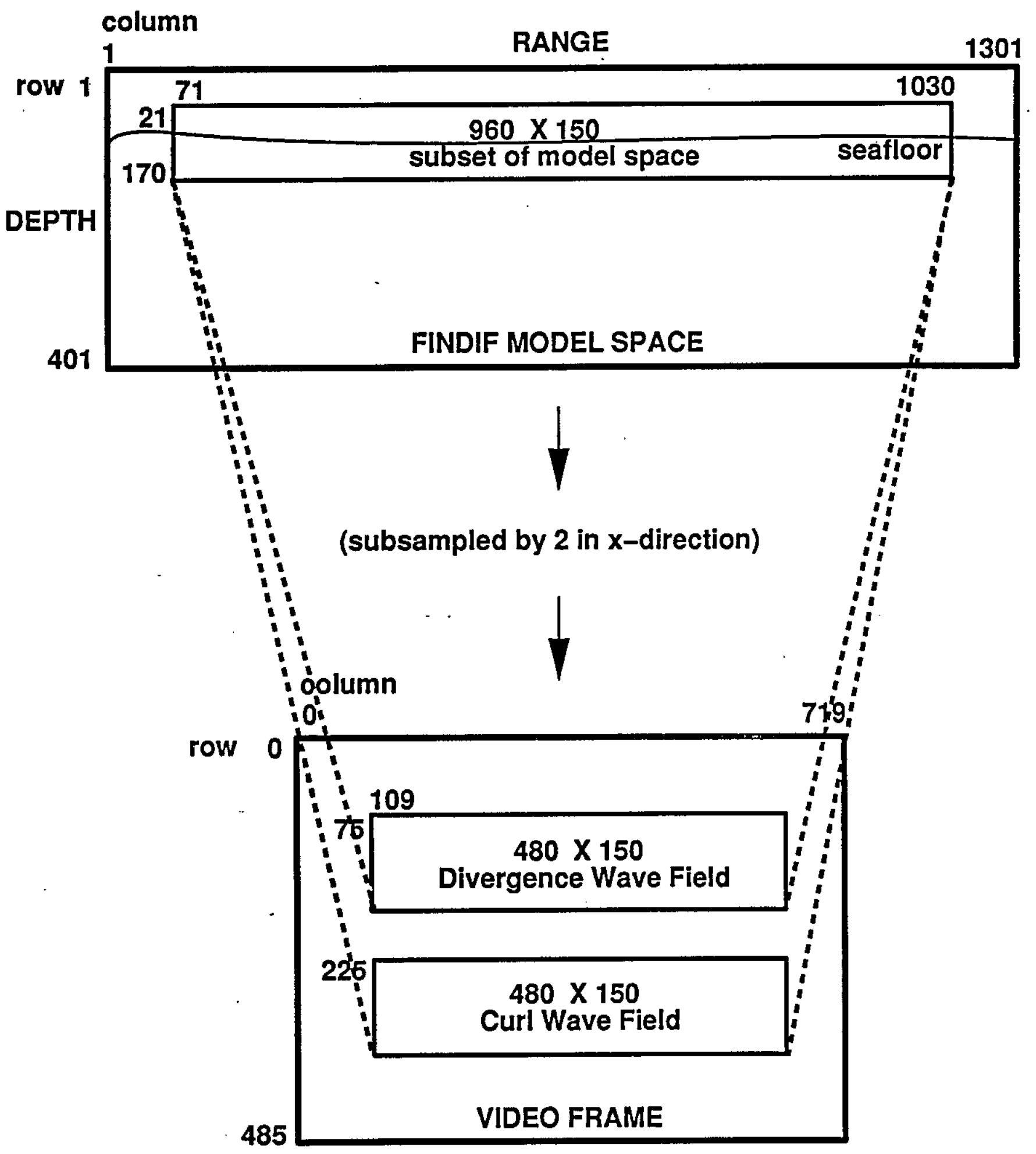




\section{PHASE 2: $\quad$ UNIX C and PHOTOSHOP Implementation}

With the return of Stephen to WHOI in 1991, a new phase of modeling and video recording began, based largely on newer faster UNIX-based scientific workstations. Many of these systems had been benchmarked by Allen using FINDIF (1991). Additionally, new commercially available software products such as Spyglass and Adobe Photoshop were making it much easier for scientists to create and manipulate computer images.

\section{Program Conversion}

As a result of these developments, it became advisable to convert AFRAME from FORTRAN language to $C$ language. It was also useful to replace the cumbersome UNIRẢS graphics subroutine calls and the lettering/line-art preprocessing script program with a more modern counterpart. Rather than use imbedded subprogram calls to yet another graphics subroutine library, it was decided instead to take advantage of the power and flexibility of Adobe Photoshop to produce all of the stationary graphics overlay information needed for each animation frame.

\section{New Software Configuration}

As a result, the new Phase 2 version of AFRAME now uses Photoshop overlay frames which are first created manually, using precisely the same format as Abekas A60 disk video frames (Figure 6). The new C-language AFRAME main program then combines these graphic overlay frames with each successive model snapshot, using simple pixel-by-pixel Boolean operations, in order to produce a final series of animation frames for the A60 video disk (Figure 7). Photoshop is also used to create a file of overlays of graphic digits which are added to the final animation frames to produce a changing frame-counter. An auxiliary program GENMSK is used to convert FINDIF boundary conditions into an overlay showing the location of the seafloor within the frame. Facility is provided for optionally using a Spyglass gray-scale or color lookup table, in order to correlate image colurs used by AFRAME displays with those used in Spyglass displays.

\section{Equipment Relocation}

The Abekas A60 disk and all of the associated hardware had been moved from the Clark Laboratory Building to the "black room" video studio in the Blake Building so that it could be more conveniently interfaced to the highly stable video "house sync" signal and WHOI's Betacam recording and editing equipment. By this point, the means of creating $\mathrm{A} 60$ frames and transferring them using ftp from VMS, UNIX, PC, and MAC computers to the A60 disk over the Internet had been perfected. The black room suite of computer equipment had grown to include at least one of each platform (except a VMS system), thereby allowing A60 users to run AFRAME on their own computer systems anywhere on the Internet (via X-windows, telnet, etc.), and then ftp their video sequences to the $\mathrm{A} 60$ directly. 


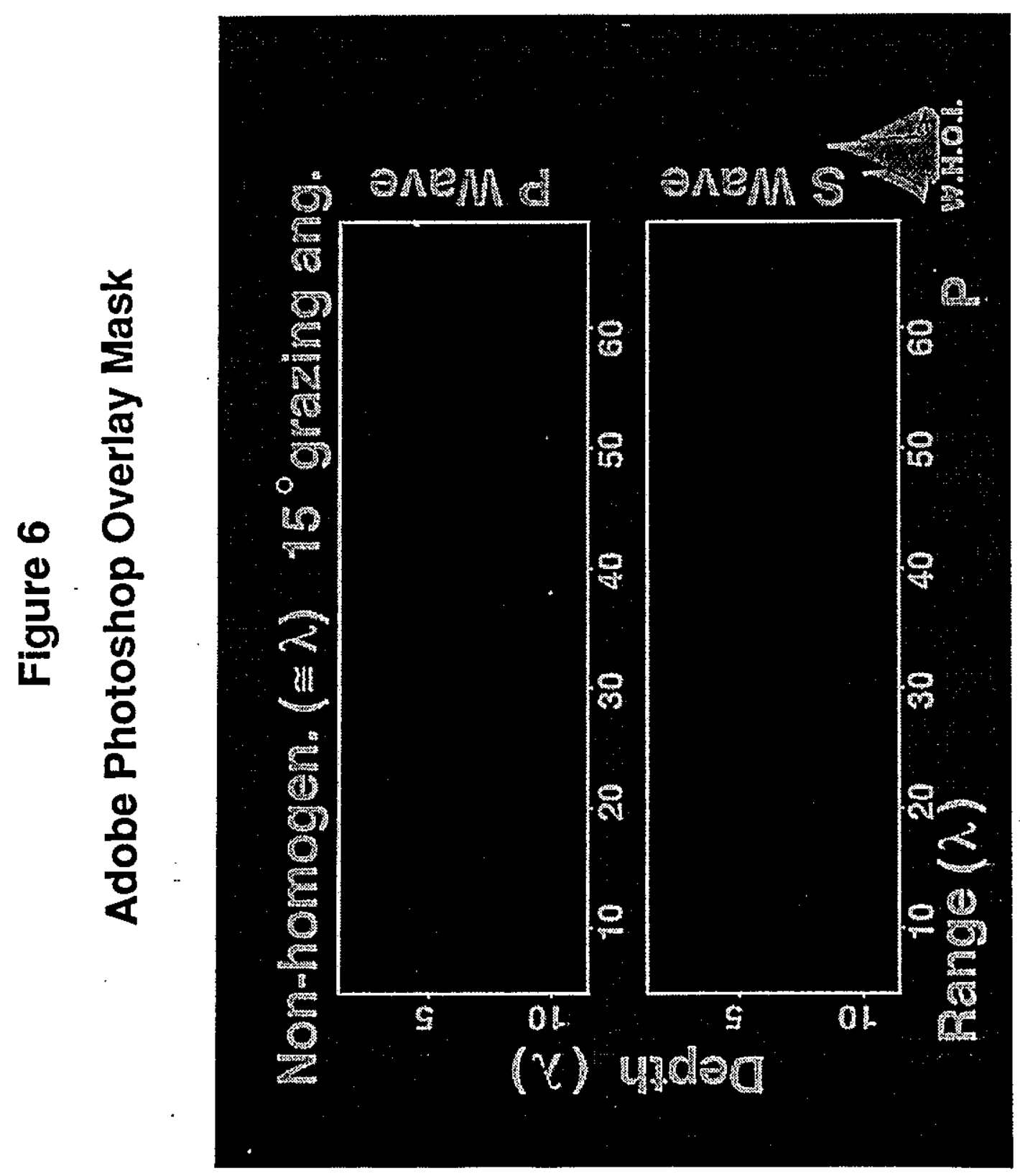


Figure 7

AFRAME Processing Flow Diagram

(Phase 2)

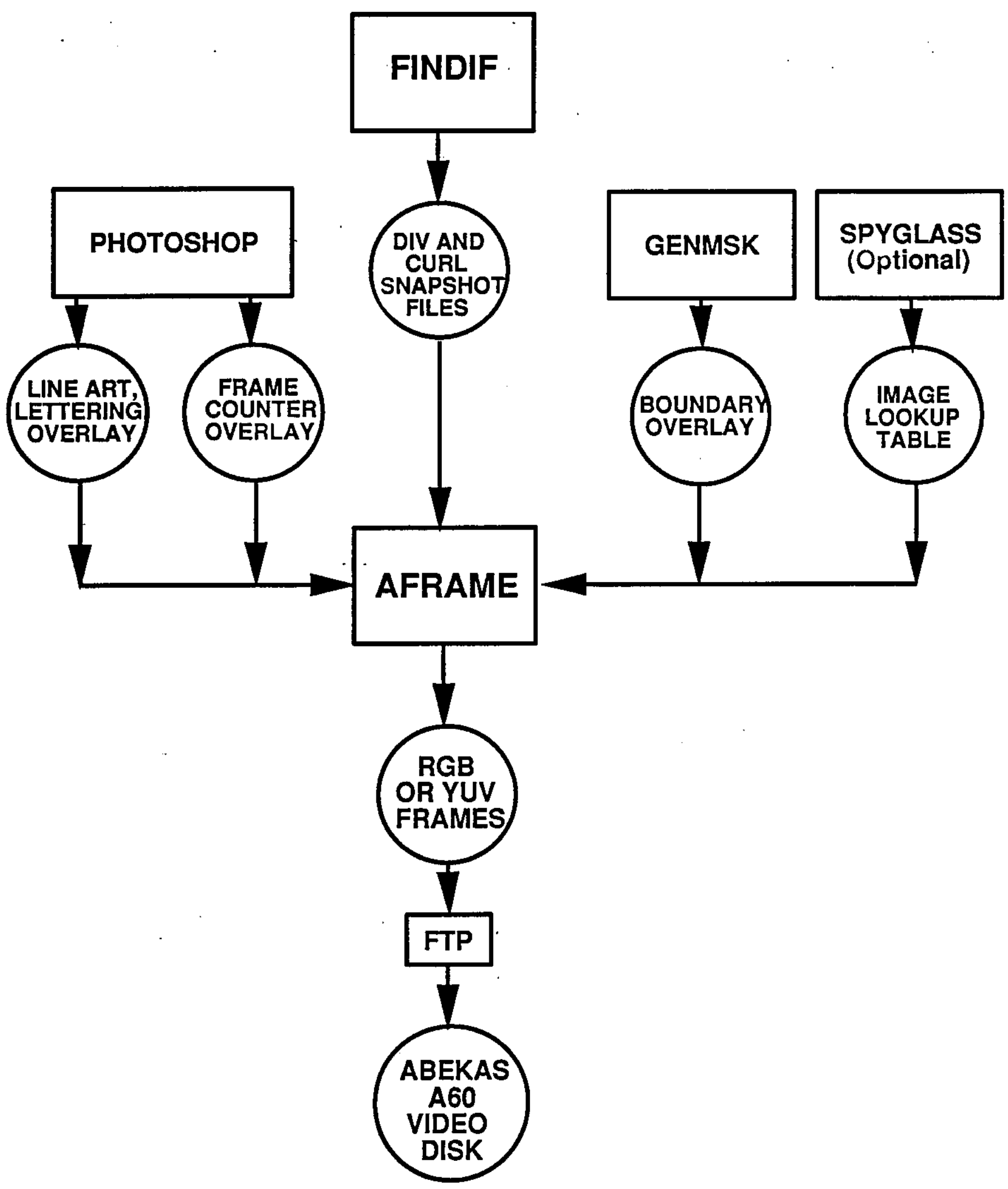




\section{Phase 2 FINDIF/AFRAME Results}

The new set-up and the new AFRAME software were used to produce a presentation video tape for the Fall, 1992 ARSRP Symposium. This tape contains a suite of four models showing the behavior of the $P$ and $S$-waves propagating through homogeneous water; a flat seafloor, a single faceted seafloor, and a canonical rough seafloor (Appendix 3).

\section{Non-homogeneous Media and Higdon Boundaries}

Further revisions of the new AFRAME were made to accommodate the model snapshots generated by Swift, et al (1995) who was using FINDIF to investigate the effects of non-homogeneities and density gradients in the subseafloor structure. Pastel color overlays were used o indicate various kinds of non-homogeneous structures and gradients in the video sequences.

Numerical analytical changes in FINDIF, particularly the introduction of new methods for handling boundary conditions (i. e., Higdon boundary code replaced Telegraph Equation boundary code), initially introduced spurious reflections and other model instabilities which were not readily apparent until they appeared in test video sequences.

The value of having moving graphical model output as a numerical debugging tool became particularly apparent during this exercise. In one case, for example, numerical noise in the wave patterns completely overshadowed the real signals. This noise might have easily been incorrectly interpreted as a meaningful model result, were it not for availability of a complete video sequence showing all the output time series data in highly condensed form. In this kind of time-varying video format, essentially all of the significant details, both desirable and undesirable, "stand out like a sore thumb." In this case, for example, the investigator is able to witness about 108 million floating point numbers in 25 seconds!

\section{Further Modifications}

In the course of this more recent work, AFRAME was yet again modified to allow for combining input from two different model runs (a) from the same or different platforms, (b) with different floating and binary representations, and (a) from UNIX and/or VMS systems. Modifications were included for using input and output frames which were compatible with Spyglass software. A processing module for the AVS scientific visualization software package was also developed, with the thought in mind that someday time-varying three dimensional graphics displays would be utilized in observing the model results from the 3-D counterpart of the FINDIF modeling program.

\section{Other Phase 2 Users}

During the second phase of the program, other investigators continued to use the A60 video disk setup, together with a variety of software tools, to produce scientific animation sequences for various presentations (Appendix 2). Of special interest is the work of $\mathrm{R}$. Signell of the Woods Hole marine 
branch of the USGS, whose widely acclaimed video of the modeling of Boston Harbor tidal flux and mixing was produced using this equipment. Signell's Cray 2 and UNIX workstation-based model output was rendered using AVS and the resulting animation sequence written directly to the $A 60$ video disk in the black room.

\section{Work in Progress}

There has been little if any development effort on this program suite for a year or so. There is still work in progress on the main AFRAME program itself, as well as on the formal program documentation. As it stands, the code is a "living program" (i.e., a program which isn't complete, and therefore can't easily be run by anybody but the author). Significant cleanup work should be undertaken in order for the program to be useful in other modeling applications or portable to other locations.

Three WHOI Technical Reports are in the early stages of preparation, one on the overall system, one on the AFRAME software, and one on system operations (i. e., a Systems Manual, a Programming Manual, and a Users Guide).

\section{Conclusion}

The implementation of the AFRAME program and the Abekas A60 video disk has provided WHOI scientists and technologists with a useful method of transferring computer graphics to broadcast quality video tape.

The advent of the MPEG data compression techniques, less expensive desktop computer-to-video recording technologies, and the growing use of Mosaic-like Internet browsers for viewing animation sequences have made the AFRAME/Abekas A60 video disk method somewhat obsolescent when it comes to publicizing time-varying computer output. For making broadcast quality Betacam master video tapes, the $A 60$ is still the way to go. This degree of quality may be "overkill" for many of today's scientific users, given the tedious nature of frame-by-frame video compositing and editing.

\section{Acknowledgment}

The author wishes to thank Dr. Ralph Stephen for his direction, scholarly guidance, and generous support of this interesting project. The author also wishes to thank Tom Bolmer, Steve Swift, Marty Dougherty, Bill Lange, and Tim Silva for their assistance with the many technical and scientific aspects of this work, and for their patience and understanding in times of "computer related emergency." Without extensive help from these individuals, along with assistance from many people in the WHOI computer center, this project would not have succeeded. 


\section{References}

Allen, J.M. , Stephen, R.A., Benchmarking the Two-Dimensional Finite Difference Synthetic Seismogram Code, W.H.O.I. Technical Report, WHOI-91-31, (September, 1991) .

Hunt, M.M., Gove, L., Stephen, R.A. , FINDIF: A Software Package to Create Synthetic Seismograms by Finite Differences, W.H.O.I. Technical Report, WHOI83-42, (November, 1983).

Kelly, K.R., Ward, R.W., Treitel, S., Alford, R.M. , Synthetic Seismograms: A Finite Difference Approach, Geophysics 41, 2-27, (February, 1976).

Little, W.S., Stephen, R.A., A Video Animation Facility for Studying Finite Difference Synthetic Seismic Wave Fields and Other Time-Varying MultiDimensional Data, Proceedings of the ARSRP Research Symposium, IGPP, Scripps Institution of Oceanography, (March, 1991).

Stephen, R.A., A Review of Finite Difference Methods for Seismo-acoustic Problems at the Seafloor. Reviews of Geophysics, 26, 445-458, (1988).

Stephen, R.A., A Numerical Scattering Chamber for Studying Low Angle Seafloor Backscatter, J. Acoust. Soc. Am. 90, 2276, (1991).

Stephen, R.A., Dougherty, M.E., Little, W.S., Finite Difference Methods for LowAngle Seafloor Scatter, Proceedings of the ARSRP Research Symposium, IGPP, Scripps Institution of Oceanography, (March, 1991).

Stephen, R.A., Swift, S.A., Modeling Seafloor Geoacoustic Interaction with a Numerical Scattering Chamber, J. Acoust. Soc. Am. 96, 973 (1994).

Swift, S.A., Little, W.S., Stephen, R.A., Signal and Noise Levels in Numerical Scattering Chamber Snapshots, W.H.O.I. Technical Memorandum, WHOI-01-95, (May, 1995). 


\section{Appendices}

Appendix 1: $\quad$ Memorandum proposing a WHOI computer animation facility 

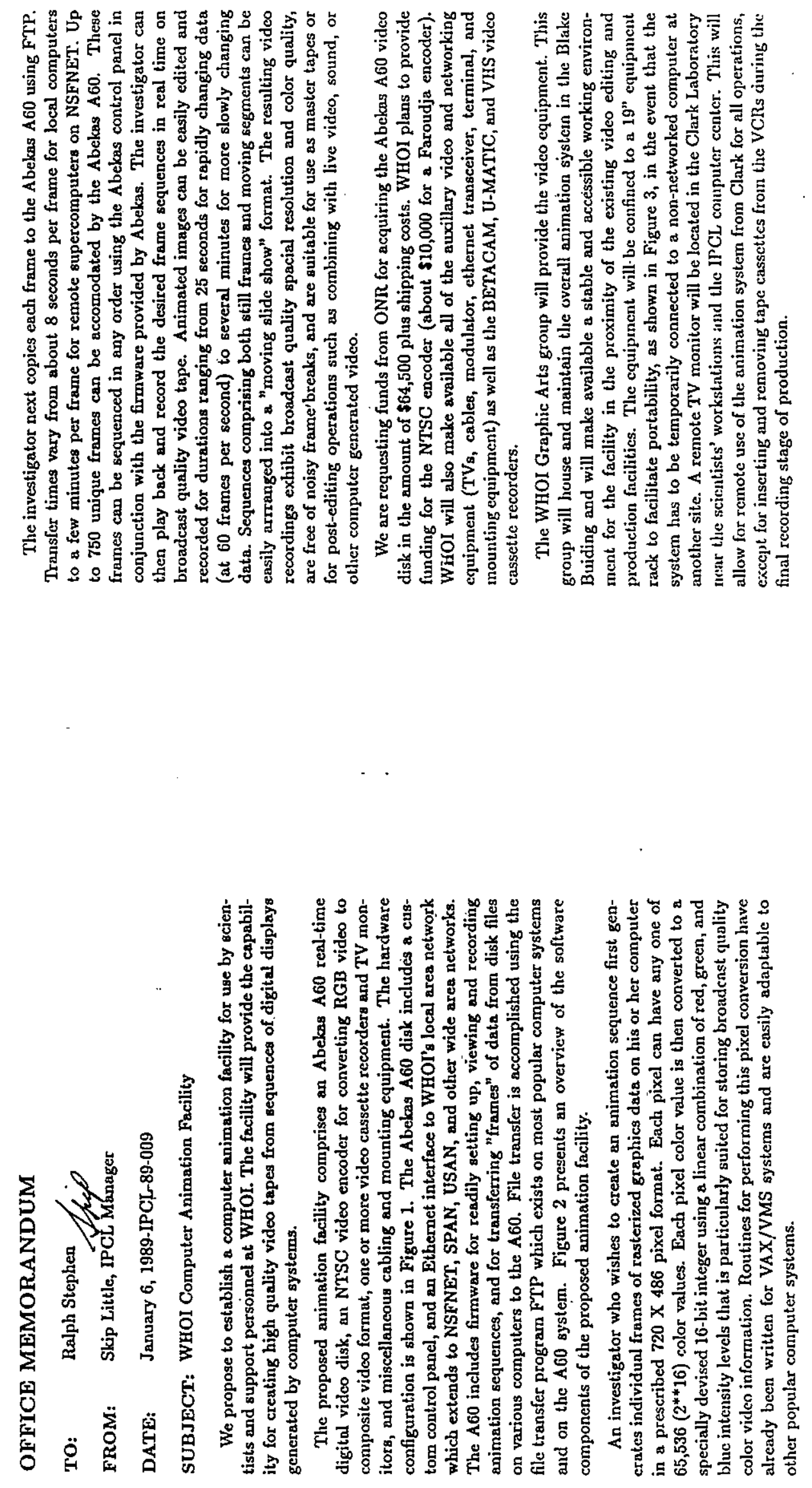
Ėgure: (ol Appendix 1)

WioI Computer Anination Facility: Hardware Configuracis:
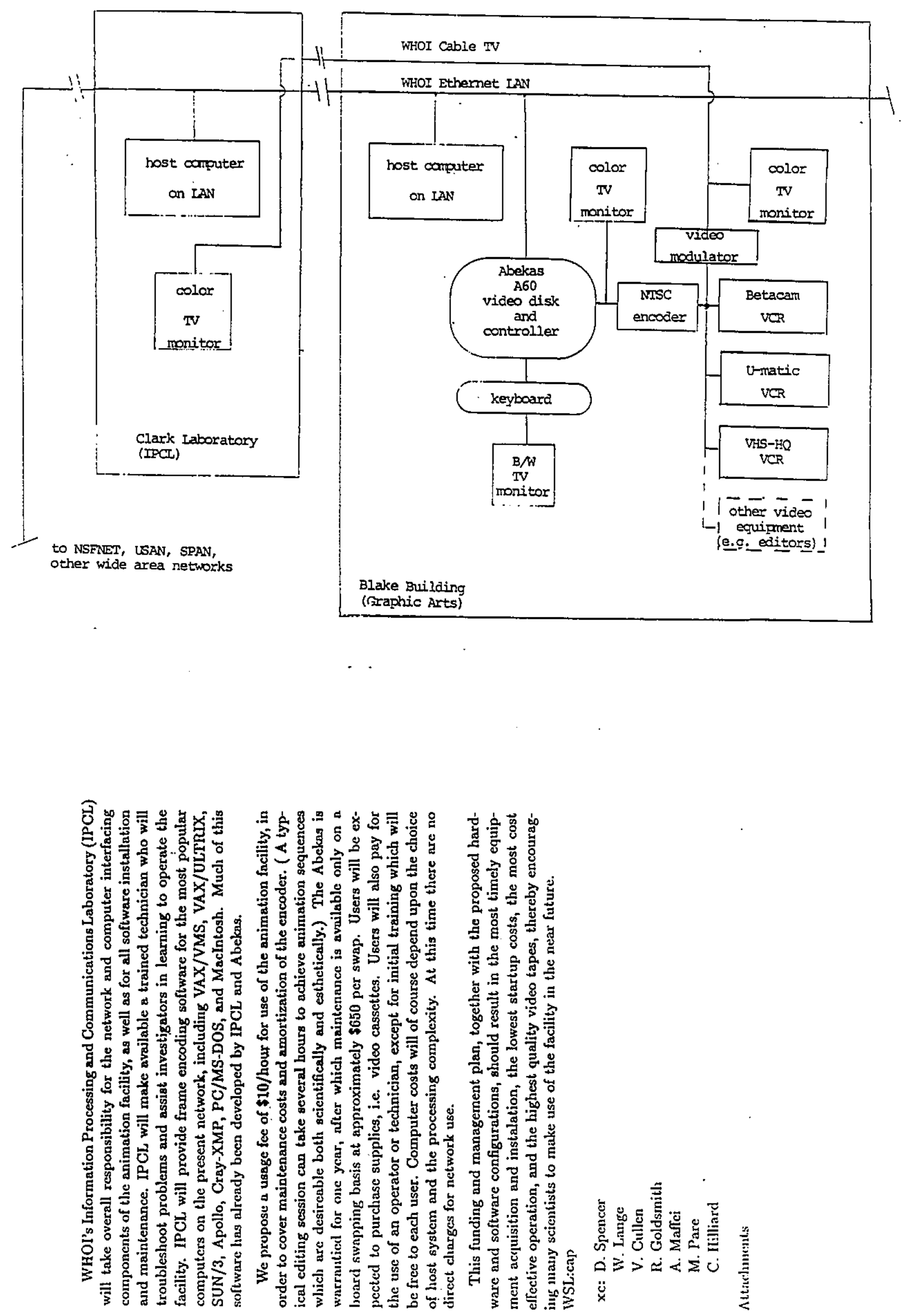

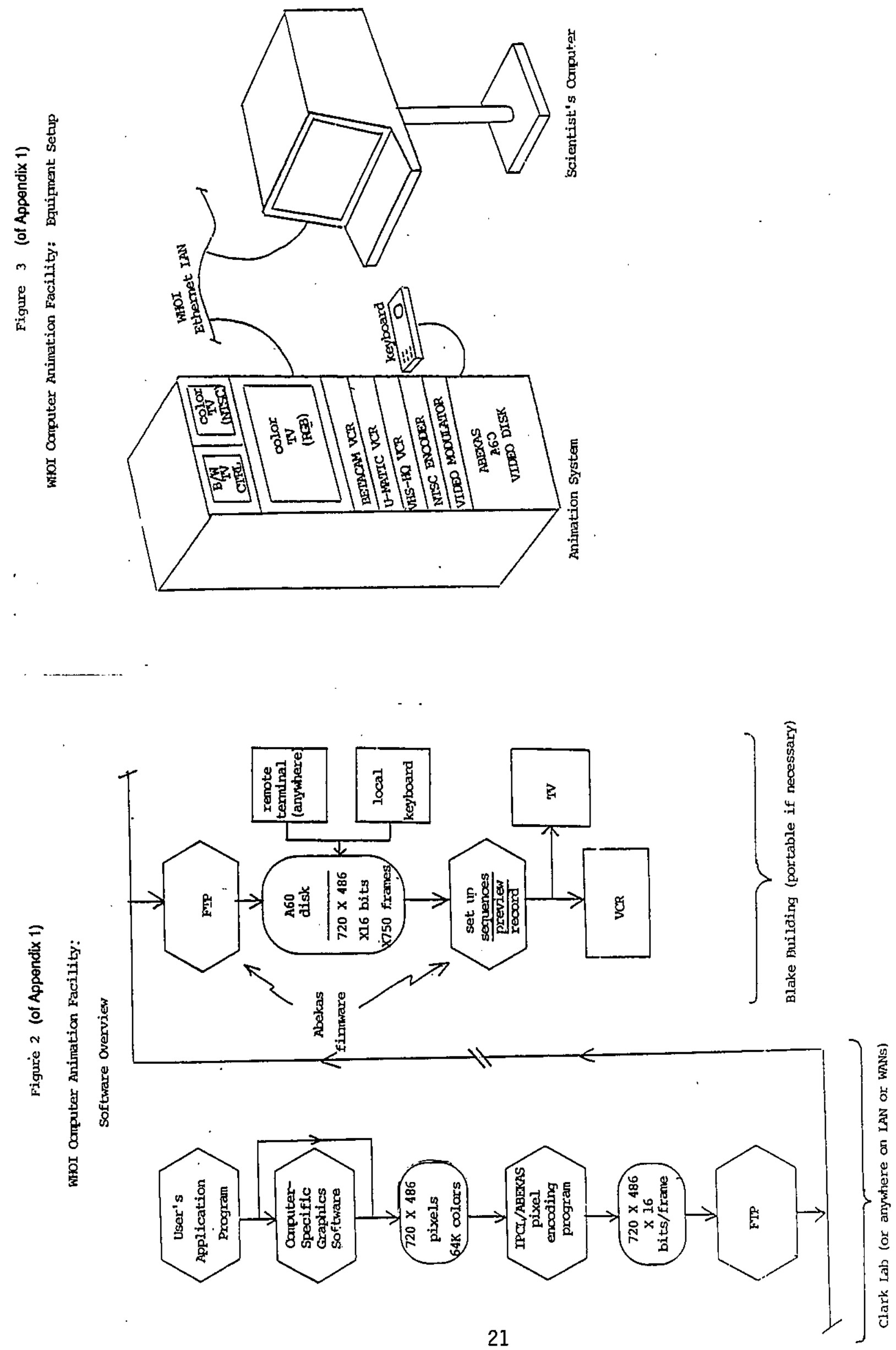


\section{Appendix 2: Selected Abekas A60 disk users}

Phase 1

Lynch, J.F., Newhall, A.E., Greenland Sea Tomography

Burns, D.R., Allen, J.M., 3-D Synthetic Seismography

Caruso, M.J, Graber, H., Jensen, R., Surface Wave Dynamics

Signell, R., Butman, B. Boston Harbor Tidal Exchange (USGS)

Phase 2

Jenkins, W.J., Tritium Distribution in the North Atlantic

Qju, B., Kelly, K.A., Joyce, T.M., Mean Flow and Variability of the

Kuroshio Extension from GEOSAT Altimeter Data

Gallo, D.G., Autonomous Benthic Explorer (ABE) animation for CNN News

\section{Appendix 3: FINDIF Video Tape Product}

Tape \#V00012, Finite Difference Synthetic Seismograms, Convex C220 runs CNT01, CNT02 (October, 1990).

Tape \#V00024, Finite Difference Synthetic Seismography, VAX 8800 runs TES12, TES13 (March, 1991).

Tape \#V00030, Oceanographic Uses of Video Animation (April, 1991).

Tape \#V00032, Selected Research Projects Utilizing Video Animation Technology (May, 1991).

Tape \#V00056, Untitled Tape for ARSRP Symposium (Hard and Soft, Flat and Rough Seafloor), runs FIV03, FIV05, FIV07, FIV08, FIV09 (September, 1991).

Tape \#V00074, Low Angle Seafloor Backscatter, runs FIB12, FIFB1, FIFB5, FIFB6 (homogeneous water, flat, faceted, and canonical rough seafloors) (September, 1992).

(Betacam master tapes, and tapes containing the results of numerical model debugging and video debugging, are not listed. ) 